\title{
The acceptability of dietary tools to improve maternal and child nutrition in Western Kenya
}

\author{
Nidal Kram ${ }^{1}$, Sarah Melgen', Ellah Kedera ${ }^{2}$, Deborah Kortso Collison', \\ Jonathan Colton ${ }^{3,4}$, Wendy Blount ${ }^{4}$, Frederick Grant ${ }^{5}$ and Amy Webb Girard $1,6, *$ \\ 'Hubert Department of Global Health, Rollins School of Public Health, Emory University, 1518 Clifton Road, Claudia \\ Nance Rollins Building, Room 7021, Atlanta, GA 30317, USA: ²PATH (Program for Appropriate Technology in \\ Health), Aphia-Plus Western, Bungoma, Kenya: ${ }^{3}$ School of Mechanical Engineering, Georgia Institute of Technology, \\ Atlanta, GA, USA: ${ }^{4}$ School of Industrial Design, Georgia Institute of Technology, Atlanta, GA, USA: ${ }^{5}$ International \\ Potato Center, Nairobi, Kenya: ${ }^{6}$ Nutrition and Health Sciences Program, Emory University, Atlanta, GA, USA
}

Submitted 16 March 2015: Final revision received 16 September 2015: Accepted 19 0ctober 2015: First published online 3 December 2015

\begin{abstract}
Objective: Dietary practices in Kenya often fail to provide adequate nutrition during the first 1000 days of life, from conception to 2 years of age. We developed and qualitatively assessed the acceptability of easy-to-use dietary tools consisting of a marked bowl, slotted spoon and illustrated counselling card to support appropriate dietary practices during pregnancy, exclusive breast-feeding and complementary feeding of children aged 6-24 months.

Design: We conducted qualitative research to assess community acceptability and obtain feedback on the design of the dietary tools.

Setting: This research took place in urban and rural communities in Western Kenya. Subjects: We conducted twelve focus group discussions with community members (mothers, husbands, mothers-in-law, community leaders) and five interviews with government nutritionists to assess acceptability and obtain recommendations on design and delivery of the tools. We conducted $24-28 \mathrm{~d}$ of user testing with fourteen pregnant women, fourteen breast-feeding women and thirty-two mothers with infants aged 6-18 months.

Results: Tools were positively received by communities. Mothers perceived improvements in their own and their children's food intakes including quantity, frequency, consistency and diversity. Many attributed perceived own and child's weight gain and/or increased energy to tool use. A minority reported using the bowl for other activities ( $n$ 9) or not using the bowl due to food insecurity $(n 5)$. Conclusions: Results suggest that such tools have the potential to positively impact maternal and child dietary practices. Future work should quantitatively assess the impact on diet and nutrition outcomes and the underlying behavioural domains associated with changes.
\end{abstract}

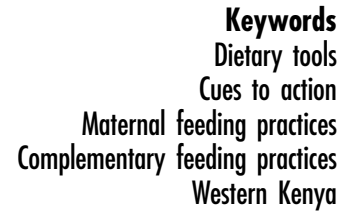

Keywords Dietary tools
Cues to action eding practices Western Kenya
Adequate nutrition during pregnancy, infancy and early childhood, also known as the first 1000 days, is critical to ensure optimal growth, development and survival of children $^{(1)}$. Sustained behaviour change within an enabling environment is foundational to dietary and nutritional improvement; thus behaviour change interventions, either on their own or in combination with other nutrition and public health interventions, are a commonly applied strategy $^{(2)}$. Recent systematic reviews of behaviour change interventions to improve infant and young child nutrition in pregnancy, exclusive breast-feeding and complementary feeding found that while practices can be improved, the degree of improvement is often small ${ }^{(3-5)}$. Systematic examination of behaviour change interventions noted that successful strategies tended to effectively use formative research to identify critical barriers to optimal practice and enablers of behaviour change and apply these findings to shape message development and optimal delivery strategies $^{(2)}$. Interventions also clearly delineated the impact pathway of the intervention, specifying the steps from behaviour change activities to nutritional impacts. However, the limited use behaviour change theory in guiding intervention design and implementation has been cited by programmers as a weakness ${ }^{(6)}$.

The Health Belief Model is a commonly used behaviour change model for preventive health strategies such as those focused on improving diets and nutrition ${ }^{(6,7)}$. This model is grounded in the idea that individuals will take up 
behaviours depending on the perceived severity of health outcomes and their perceived susceptibility to these outcomes, the perceived benefits of adopting the behaviours to prevent those outcomes and whether these outweigh perceived barriers to adoption and maintenance. Individuals' self-efficacy, or belief in one's ability to adopt and maintain a behaviour change, is increasingly identified as one of the most critical and predictive components of the Health Belief Model ${ }^{(8)}$. Lastly, cues to action are a central tenet of the Health Belief Model as they trigger individuals' actions towards the desired health behaviour and may support adoption or maintenance of a behaviour. Cues can be either external, such as media campaigns, change agents or reminders, or internal, for example perceived positive or negative changes in a person's physical or emotional well-being ${ }^{(8)}$. They may be especially powerful tools if they reinforce other domains of the behaviour change model, for example beliefs in benefits of the behaviour change through perceived changes in physical well-being or enhanced self-efficacy to achieve the behaviour change. To date, however, cues to action are considered the least developed domain of the Health Belief Model $^{(8)}$.

Programmatic and research experiences highlight that families often lack clarity on the details of how to adequately nourish themselves and their children or may face socio-cultural and economic barriers to optimal feeding that they perceive are insurmountable ${ }^{(3-5,9,10)}$. They may underestimate how much and how often to feed very young children, how thick complementary foods should be to ensure optimal energy density or how much extra food is needed by women to support pregnancy and breast-feeding. Perceptions regarding what types of foods are appropriate and safe for young children, pregnant and lactating women may result in nutrient-dense foods such as eggs, meat, legumes and certain fruits and vegetables being restricted in these groups ${ }^{(9,11,12)}$; similarly, families may delay thick foods for children due to fears of choking. It is thus not surprising that a major challenge recently documented for behaviour change communication programmes for maternal and child nutrition is 'translating generic guidelines into locally appropriate recommendations and messages ${ }^{,(6)}$. As a result, the quantity, quality and number of meals during these critical periods often remain below recommendations ${ }^{(13)}$. These issues likely explain why childhood malnutrition remains a major global health problem contributing to nearly $3 \cdot 1$ million child deaths each year ${ }^{(11,14)}$.

In the context of the Health Belief Model, child feeding bowls have been identified as one type of cue to action that may trigger and support optimal complementary feeding of children aged 6-24 months. The Manoff Group developed a child feeding bowl that uses demarcations to cue meal volume. This tool was found to be acceptable among families in Latin America but the effects on dietary practices, including shifts in meal volume, have not been reported $^{(15)}$. It is unclear whether these bowls can be extended to cue other domains of optimal complementary feeding such as meal frequency, diversity or thickness; or whether they can be expanded to include the first 500 days of life when support for mothers' nutrition during pregnancy and the period of exclusive breast-feeding is paramount.

Building on the Health Belief Model and more specifically the concept of cues to action, we developed the 1000 days dietary tools to be used by families to promote not only optimal meal volume, but also meal frequency, consistency and diversity from pregnancy to 2 years of life. These tools include a bowl with demarcations and symbols that cue meal volume and frequency from pregnancy to 24 months of age, a slotted spoon to cue consistency of complementary foods and a counselling card that illustrates use of the tools to achieve optimal nutrition as well as diet diversity and hygiene. We hypothesize that the 1000 days dietary tools will be acceptable and easy to use, that they will simplify nutrition recommendations and that they will support mothers' efforts to improve their own and their infants' dietary practices.

\section{Methods}

\section{Concept development}

The tools were envisioned to be used as a set by families to cue adequate intakes throughout the entire 1000 days. To achieve this goal, the tools would ideally be provided to women in early pregnancy and use continued until the child was $>24$ months of age. The underlying commonality in the narrative accompanying the tools is the emphasis that the food in the bowl feeds the baby, ideally with this narrative emphasized in counselling that would accompany the toolkit. For example, in pregnancy the demarcation on the bowl and images in the counselling card would cue the woman to take her normal diet for herself, plus one additional serving of nutritious food from the bowl each day 'for the baby' growing in her belly. The mother would continue to use the bowl in the same way during the first 6 months postpartum to support her diet during the period of exclusive breast-feeding. The mother then transitions the bowl to the infant at 6 months to facilitate timely introduction and age-appropriate meal frequency and quantity. As the child ages, the mother gradually increases the amount and frequency of foods as indicated by the lines and symbols on the bowl. The slotted spoon allows the mother to test the consistency of the infant porridge after it has been cooled to an acceptable temperature to ensure it is of adequate thickness for the child. For example, if porridge flows through the slots, the mother knows the porridge is too thin and would be cued to add locally available ingredients to thicken it. The spoon's function is crucial to promoting the perception that volume of food alone does not translate to adequate nutrition and that food thickness can better ensure a more appropriate 
nutrition-to-volume ratio. The counselling card provides pictorial instructions on recommended feeding practices through the entire 1000 days including diet during pregnancy, exclusive breast-feeding for the first 6 months and complementary feeding practices from 6 to 24 months, and includes pictures showing how to use the bowl and spoon to achieve these recommendations. The card also provides pictorial information on dietary diversity, including the types of locally available nutrient-dense foods to add to porridge to improve its thickness and nutritional value. Optimal hygiene practices including hand washing, safe water and food preparation, and cleaning of the bowl and spoon are also represented.

\section{Development of the tools}

Bowl prototypes were developed using the general programmatic recommendation of one extra meal per day ( 1675-2090 extra kilojoules ( 400-500 extra kilocalories)) for women during pregnancy and breast-feeding ${ }^{(16)}$ and the guidelines of Dewey and Brown for adequate meal volume and frequency for breast-fed infants (6-23 months of age), assuming average breast-feeding and medium energy density for complementary foods ${ }^{(17)}$. The mediumenergy-density estimation was used because the spoon would prevent the provision of low-energy-density/thin foods. The authors of the present study determined the spoon slot size using the thicknesses of locally relevant complementary feeding porridges (i.e. maize porridge, known as uji) of varying energy densities. The slot size deemed most appropriate for ensuring adequate thickness was $0 \cdot 3 \mathrm{~cm}$. Multiple prototypes of the bowl were rendered using SolidWorks, a three-dimensional modelling program, and printed on a 3D Stratasys Dimension sst1200es printer (Eden Prairie, MN, USA). Preliminary research and refinements to the bowl, spoon and counselling card were made based on inputs from Sudanese and Ethiopian mothers with young children and with maternal and child health and behaviour change communication experts at CARE and Emory University (Atlanta, GA, USA). The final prototypes of the bowl and spoon used in Western Kenya were produced in foodgrade polypropylene via a protomold ${ }^{\circledR}$ injection moulding process by Proto Labs, Inc. (Maple Plain, MN, USA). The counselling card messages and images were adapted from the nutrition and infant and young child feeding counselling materials used in government hospitals in Kenya. Images, design and costing details of the tools are available from the authors upon request.

\section{Design, study site and sampling}

We conducted qualitative research to test the feasibility and acceptability of the tools to promote optimal dietary intake of mothers during pregnancy, of mothers during the first 6 months postpartum, as well as of children aged 6-18 months in one urban and one rural community in Western Kenya. Although estimates are not currently available by Province, in 2014, $26 \%$ of Kenyan children aged 6-23 months were classified as malnourished; $61 \%$ of infants aged 6 months or younger were exclusively breast-fed and only $21 \%$ of children aged 6-23 months received a minimally adequate diet in terms of breastfeeding, frequency and diversity ${ }^{(18)}$. Maternal malnutrition is also a challenge in Kenya ${ }^{(19)}$. Twenty per cent of women aged $15-45$ years have a BMI of $<18.5 \mathrm{~kg} / \mathrm{m}^{2(20)}$, and the prevalence of anaemia among pregnant women in Kenya is as high as $70 \%$ in some areas ${ }^{(21)}$.

The selected communities were the sites of formative and pilot work for an integrated health, nutrition and agriculture project implemented in Bungoma and Busia Counties. At the time of the present study, pilot activities had ceased in these communities. Participants in focus group discussions (FGD) and user testing were mobilized and purposively sampled by a community liaison with over a decade of experience working in the communities and with the assistance of community health workers who had worked with the community for three or more years. Participants were selected based on their residence (urban $v$. rural) pregnancy/lactation status and the age of their infants. Participants were selected to be representative of the larger communities as opposed to extreme cases.

\section{Data collection}

A team of six local research assistants, with university degrees in nutrition and trained by the authors in qualitative research methods, research ethics and participatory counselling using the tools, conducted all data collection and counselling. To assess community acceptability and obtain feedback on the bowl, spoon and counselling card, research assistants conducted a total of twelve FGD (six urban and six rural) with pregnant women, women 0-24 months postpartum, mothers-in-law, community leaders, husbands and frontline health workers. FGD were conducted in Kiswahili with six to ten participants per group (106 participants in total) and lasted approximately 60 min. FGD explored current norms for maternal and infant dietary practices and awareness of, opportunities for and barriers to recommended practices. Following this, discussion groups were introduced to the counselling card and the bowl and spoon and queried regarding ease of understanding, use, acceptability and feasible delivery platforms. The first and second authors additionally conducted five in-depth interviews in English with the District Nutrition Officer, two government nutritionists and two nutrition advisors who specialize in maternal and child nutrition to understand the relevance of the tools for ongoing activities and their acceptability and feasibility in Western Kenya.

User testing was conducted using a trial of improved practices methodology with sixty women (thirty urban and thirty rural) representing the three critical periods of the first 1000 days: pregnancy, exclusive breast-feeding and 
complementary feeding. Women who participated in user testing were not the same as those who participated in community FGD. Women used the tools over a $28 \mathrm{~d}$ period and were interviewed three times over this period. At baseline, research assistants interviewed mothers about their and their children's (if relevant) usual dietary practices, including typical composition of meals, frequency of meals and snacks, and typical quantity of foods consumed at each meal. The research assistants introduced the tools and provided instructions on their use; other family members were invited to participate in this counselling session. Mothers and others present were queried about their expectations of the tools and potential challenges they might face in their use. Research assistants addressed any concerns or questions raised about the tools. Mothers were then engaged in dialogue and provided with ageappropriate dietary counselling based on current practices and constraints, including the use of locally available, nutrient-dense foods that could be used to thicken infant porridge. Approximately $12-14 \mathrm{~d}$ later (midline) mothers were interviewed about their own and/or their infants' dietary practices since baseline and their experiences with the tools. Research assistants engaged in additional clarification and counselling, as needed.

Approximately 12-14d later (endline), mothers were interviewed about dietary practices since midline; additional open-ended questions queried participants' overall experiences with the tools, acceptability, challenges, potential delivery platforms and recommendations for improvements. Two weeks following user testing, we conducted two FGD, one with ten rural mothers and one with six urban mothers who participated in user testing to further discuss collective experiences using the tools and gather opinions about acceptability, feasibility and potential delivery strategies.

\section{Data management and analysis}

All FGD and in-depth interviews were recorded with participants' permission. We prepared detailed summaries of FGD and verbatim transcripts of key informant in-depth interviews. All interviews during user testing were recorded with participant permission and recordings were used to clarify responses to open-ended questions in the questionnaire. Detailed summaries, transcripts and openended responses were analysed in the qualitative data analysis software MAXQDA 11 using a thematic analysis approach to identify common dietary practices, beliefs, barriers and enablers to optimal practices and understand community perceptions of the feeding tools and recommendations for changes. Responses of participants in user testing on their perceptions of the tools as well as perceived changes in dietary practices at baseline, midline and endline were analysed using Microsoft ${ }^{\circledR}$ Excel version 15. Columns were created for each question in the interview guide and all answers were numerically coded and entered for each participant based on her answer.
It should be noted that research assistants did not quantitatively assess the amounts of foods consumed by mothers or their children. Rather, they approximated the amount of food using the demarcations on the bowl. Those consuming at or above the demarcation for their life stage were considered to meet recommended intakes. Consistency of foods was based on observation where possible and on the mother's description of the thickness of the porridge and whether it flowed through the slots in the spoon.

\section{Results}

\section{Community norms regarding maternal and child feeding practices}

Participants reported that women usually consumed tea, two meals and two snacks per day. Meals consisted of ugali, a dense boiled corn porridge, accompanied by vegetables (such as kale, amaranth leaves and jute plant), legumes (kidney beans or cowpeas) and fish; snacks were fruits or peanuts. Breast-feeding women reported consuming an extra meal each day because they are expected to do so by their mothers-in-law and indicated the extra meal was to increase milk production. However, consumption varied by season, cultural beliefs about the effects of different foods on milk production, size of the baby at delivery and a family's purchasing power. For example, breast-feeding women indicated that cowpea leaves prepared with bicarbonate and other spices should be avoided as the bicarbonate and spices are believed to reduce milk production. Conversely, pregnant women are advised to consume less food during pregnancy and avoid foods like avocado, plantains and particular types of fish. It is believed that these foods increase the baby's size resulting in birth complications. Maternal diet patterns did not vary across the urban and rural communities.

For infants, timing of introduction of complementary foods varied by family finances and infants' perceived level of dissatisfaction with breast milk alone. Figure 1 illustrates foods identified by respondents as commonly fed complementary foods and the times at which these different foods are introduced. Infants are typically breast-fed

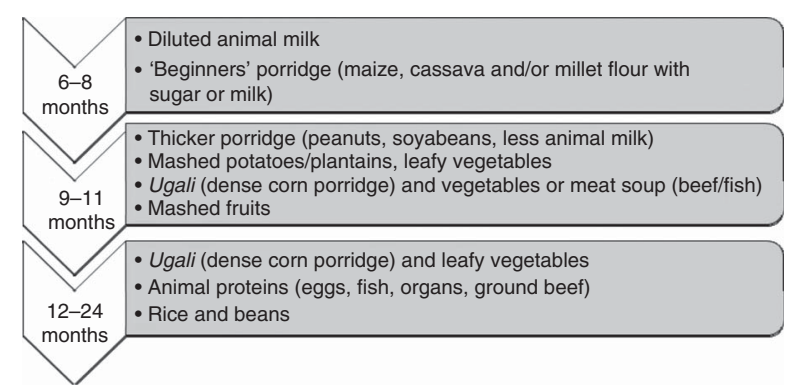

Fig. 1 Commonly fed complementary foods and initiation times for children aged 6-24 months in Western Kenya 
although those younger than 8 months may be introduced to diluted cow's milk, water and thin 'beginners' porridge (uji) as early as 2 months; by 8 months of age they are regularly fed breast milk, diluted cow's milk and water, and thin $u j i$ two to three times per day. Thicker foods are introduced after the age of 8 months. Many nutrientdense foods, such as meat and legumes, are introduced to children between the ages of 12 and 24 months due to fears of choking. Eggs, although highly nutritious, are not fed to younger children ( $<12$ months) because of the common belief that consumption would delay speech development. Forced feeding was cited as a common practice, especially when children were ill and refused to eat.

\section{Self-reported effects of tools on dietary practices}

Fourteen pregnant women (seven urban, seven rural), fourteen women 0-6 months postpartum (seven urban, seven rural) and thirty-two women including three mothers with twin children aged 6-18 months (sixteen urban, sixteen rural) participated in user testing. Of the sixty participants, nine did not complete all three interviews. At baseline, only $28 \%$ (4/14) of pregnant women reported consuming three to four meals per day excluding snacks (Table 1). By midline all pregnant women had increased the number of meals consumed by at least one extra meal per day and this practice was sustained through endline (Table 1). Pregnant women also reported perceived increases in energy, weight gain and reduction in headaches. An urban pregnant woman said:

'I take a bowl full of githeri [a mixture of beans and corn] or banana [cooked plantains] [as a normal meal] then I take porridge afterwards [as an extra meal]. I now have strength in the body to be able to work. I used to eat [two meals] before [the study] but I would still feel weak [after the two meals]. But I now have strength because of the extra porridge that I take after eating [two meals].'

Breast-feeding women reported similar changes in their diets to pregnant women. Breast-feeding women also reported a combination of perceived benefits including increased milk production, own weight gain, increased energy and infant weight gain. A rural breast-feeding woman said: 'I feel it in my body and people are also saying that I am fatter.' An urban mother of a 1-month-old perceived her child gained weight:

'...one of my friends who has a baby the same age as mine asked me if I had gone to the medicine men to buy the drug [that] makes children grow fat quickly... The [friend's] baby was big before, but now my baby is bigger than hers and she was wondering what I did... I told her to eat well and just breast-feed the baby until six months and the baby will be big.'

These benefits appeared to influence women's decisions to supplement or continue to exclusively breast-feed. For example, three breast-feeding mothers stated at the baseline visit that they intended to give supplemental foods before 6 months due to perceived 'lack of milk'. However, after using the tools to support their own diet, they chose to continue exclusive breast-feeding to 6 months. The following conversation with an urban breast-feeding woman illustrates this:

Interviewer: 'You said at the moment you have a lot of milk, what could be the reason for that change?'

Mother: 'The food that I eat [now]. Before you visited, I never use to take that extra portion. But since I started taking another extra portion, I have milk.'

Interviewer: 'Previously you said that when the baby reaches $3 \frac{1}{2}$ months you will introduce other foods, are you are still going to do that?'

Mother: 'He will breast-feed until 6 months.'

Interviewer: 'Why have you decided that?'

Mother: 'I never use to have milk but now the baby suckles well and there is a lot of milk.'

Use of the tools was not without challenges. The two most commonly reported challenges among pregnant women included cultural beliefs related to eating less food during pregnancy and financial constraints that limit access to food. Thirty-five per cent (5/14) of pregnant women at baseline stated fear of having a large baby and the inability to have a natural delivery due to consumption

Table 1 Changes in nutritional practices among pregnant and breast-feeding women, by self-report, at baseline, midline and endline in Western Kenya

\begin{tabular}{|c|c|c|c|c|c|c|c|c|c|c|c|c|}
\hline \multirow[b]{3}{*}{ Women's status } & \multicolumn{6}{|c|}{ Consume three or four meals per day } & \multicolumn{6}{|c|}{ Consume an extra meal per day } \\
\hline & \multicolumn{2}{|c|}{ Baseline } & \multicolumn{2}{|c|}{ Midline } & \multicolumn{2}{|c|}{ Endline } & \multicolumn{2}{|c|}{ Baseline } & \multicolumn{2}{|c|}{ Midline } & \multicolumn{2}{|c|}{ Endline } \\
\hline & $n / N$ & $\%$ & $n / N$ & $\%$ & $n / N$ & $\%$ & $n / N$ & $\%$ & $n / N$ & $\%$ & $n / N$ & $\%$ \\
\hline $\begin{array}{l}\text { Pregnant women } \\
\text { Breast-feeding women 0-6 months postpartum }\end{array}$ & $4 / 14$ & 28 & $\begin{array}{l}12 / 12 \\
14 / 15\end{array}$ & $\begin{array}{r}100 \\
93\end{array}$ & $10 / 10$ & $\begin{array}{r}100 \\
85\end{array}$ & 0 & 0 & $\begin{array}{l}10 / 12 \\
13 / 15\end{array}$ & 83 & $9 / 10$ & 90 \\
\hline Breast-feeding women 0-6 months postpartum & $6 / 15$ & 40 & $14 / 15$ & 93 & $12 / 14$ & 85 & $6 / 15$ & 40 & 13/15 & 86 & $12 / 14$ & 85 \\
\hline
\end{tabular}


of too much foods or fatty foods. The most commonly reported challenge by breast-feeding women during the user testing period was food insecurity, as mothers were expected to eat last and often went without food in times of food scarcity. An urban breast-feeding woman said:

'If there is no food to add I do not use it [the bowl]. I mean in the days to come if I will not have enough food I will not add an extra portion of food.'

Another woman said:

'You said that we should exclusively breast-feed our babies for 6 months but sometimes we lack food and we do not have enough milk for the baby. Another thing is that sometimes food is little so one cannot measure the amount that you told us.'

At the endline interview three breast-feeding women reported a decrease in their use of the tools due to a lack of food.

Mothers indicated that complementary feeding practices of infants aged 6-18 months improved over the course of the study (Table 2); mothers reported that they increased the frequency, amount and thickness of foods given to their children. Mothers also reported increasing the diversity of food and introducing new foods like leafy vegetables, beans, fish and eggs.

'There are different types of food that I give him. For instance when my husband was leaving today, I asked him to come [home] with some potatoes for him [the baby]. I also boil eggs for him [the baby]. I told my husband that the foods on the [counselling] card are the ones recommended for our child, so he works hard to get him [the baby] some [recommended foods] every day.' (Rural mother of a 14-month-old)

By endline, $79 \%$ (23/29) of mothers perceived their children had gained weight, had increased energy and/or had reduced illness; they attributed these changes to changes in feeding practices. Three women who indicated their children were malnourished at baseline reported perceived improvements in children's weight at the end of the user testing period; however, these changes were not confirmed with anthropometry. As one urban mother said: 'She has gained weight... I think I was giving her less food before.'

Mothers overwhelmingly expressed appreciation for the bowl and said it gave them confidence in their ability to feed the appropriate amount and frequency of meals:

'For me it was the demarcations on the bowl that pleased me as they enabled me to feed my baby food that was adequate for his stomach since before then I didn't feed enough food, but when you gave me the bowl I now see that the baby has good health.' (Rural mother of a 10-month-old)

'Before [receiving the tools] I couldn't monitor the amount of food that I was feeding him. Sometimes I would think that a lot of ugali would cause his stomach to swell so I just feed him a small amount. When he refused to take his porridge, I would immediately give it to the other children. Currently, I know the amount of food that is recommended for him and I am certain that he gets satisfied. The bowl gives me the recommended amount of food that I am supposed to feed to my baby. Maybe before I used to either overfeed him or underfeed him but this bowl has been of great help and that is why I insist that I cannot allow you to take it away from me!' (Rural mother of a 7-month-old)

Food insecurity was not reported by mothers as a challenge for increasing the amount of food fed to infants. Rather, challenges to using the tools for complementary feeding were related to misperceptions about the types of foods infants could eat. At the beginning of user testing, mothers were uncomfortable with incorporating nutrientdense foods, especially animal-source foods, into their children's diets. As a result very few mothers were providing these foods at baseline. Mothers, mainly those with children aged 6-12 months, were afraid of choking the child, while others refused to feed foods like eggs because of beliefs about adverse consequences. Research assistants counselled mothers on the importance of food diversity and methods of food preparation to offset food

Table 2 Complementary feeding practices, by mothers' report, of children aged 6-18 months at baseline, midline and endline in Western Kenya $(n$ 35)

\begin{tabular}{|c|c|c|c|c|c|c|c|c|c|c|c|c|c|c|c|c|c|c|}
\hline \multirow[b]{3}{*}{ Child's age (months) } & \multicolumn{6}{|c|}{$\begin{array}{l}\text { Consuming recommended number of } \\
\text { semi-solid meals per day }\end{array}$} & \multicolumn{6}{|c|}{$\begin{array}{l}\text { Consuming recommended amount of } \\
\text { semi-solid food during meals }\end{array}$} & \multicolumn{6}{|c|}{$\begin{array}{l}\text { Consuming recommended } \\
\text { thickness of porridge }\end{array}$} \\
\hline & \multicolumn{2}{|c|}{ Baseline } & \multicolumn{2}{|c|}{ Midline } & \multicolumn{2}{|c|}{ Endline } & \multicolumn{2}{|c|}{ Baseline } & \multicolumn{2}{|c|}{ Midline } & \multicolumn{2}{|c|}{ Endline } & \multicolumn{2}{|c|}{ Baseline } & \multicolumn{2}{|c|}{ Midline } & \multicolumn{2}{|c|}{ Endline } \\
\hline & $n / N$ & $\%$ & $n / N$ & $\%$ & $n / N$ & $\%$ & $n / N$ & $\%$ & $n / N$ & $\%$ & $n / N$ & $\%$ & $n / N$ & $\%$ & $n / N$ & $\%$ & $n / N$ & $\%$ \\
\hline $6-\varepsilon$ & $4 / 14$ & 28 & $10 / 12$ & 83 & $12 / 12$ & 100 & $6 / 14$ & 42 & $11 / 12$ & 91 & $11 / 12$ & 91 & $3 / 14$ & 21 & $10 / 12$ & 83 & $10 / 12$ & 83 \\
\hline $9-11$ & $7 / 10$ & 70 & $7 / 9$ & 77 & $6 / 7$ & 85 & $1 / 10$ & 10 & $8 / 9$ & 88 & $7 / 7$ & 100 & $7 / 10$ & 70 & $7 / 9$ & 78 & $6 / 7$ & 85 \\
\hline $12-18$ & $5 / 11$ & 45 & $4 / 10$ & 40 & $8 / 10$ & 80 & $0 / 11$ & 0 & $5 / 10^{*}$ & 50 & $5 / 10^{*}$ & 50 & $5 / 11$ & 45 & $4 / 10$ & 40 & $7 / 10$ & 70 \\
\hline
\end{tabular}

*Depicts lower uptake; mothers of 12-18-month-olds used the bowl for ugali (dense corn porridge) only and did not measure accompaniments (vegetables, meat/eggs) even though they were consumed. 
taboos. By endline nearly all mothers of 6-12-month-olds were giving their infants eggs, beans and/or some form of meat. Among women with children aged 12-18 months, the number providing animal-source foods nearly doubled from baseline to endline:

'I thought that their speech development would be delayed by introduction to [of] eggs but I hear them saying "ma... ma... ma." They are so happy. When you just place them on the ground, they start moving their feet. They just want to walk.' (Urban mother of 7-month-old twins)

\section{Mothers' perceptions of family influences on maternal and child nutrition practices and tool use} Mothers in both user testing and focus groups reported that their dietary practices and their infant feeding decisions were often dictated by other family members including husbands and mothers-in-law. A rural mother of a 4-month-old said:

'I always hear on the radio that we should not give the baby porridge, but whenever my mother-in-law hears the baby crying, she tells me to make the baby porridge. My husband said I shouldn't so I have not given the baby porridge...'

Interviewer: 'If your husband told you to give the baby food, would you do it?'

Mother: 'I will just have to do it.'

Women indicated that the tools cued other family members to support their or their infant's nutrition. An urban pregnant woman said:

'He saw the [counseling] card and realized the kind of foods that pregnant women are supposed to eat like carrots, fish, ugali, porridge, eggs; so he buys fish at least once in two weeks even though it is expensive.'

A mother of 13-month-old twins said:

'They [the family] are so excited about them [the tools]. You see, my mother-in-law says that they [the tools] are made of durable material and she even wishes that she could get one for herself.'

In the few cases where family members were not in support of the tools, it was largely because of food insecurity. A rural breast-feeding mother explained the conflicting opinions of her husband and her mother-in-law, she said:

'He told me to use them... [my mother in-law] asked me, "Where will I get all those foods?", but I told her that I will use the bowl and I will eat the food that I find.'

\section{Acceptability of tools}

The bowl, spoon and counselling card were well received. Focus group participants (community health workers, fathers, mothers-in-law, pregnant and lactating women) and key informant interviewees appreciated the durability of the bowl and spoon material; most liked was the concept of the slotted spoon to measure the thickness of children's porridge and the messages on the counselling card as they were clearly relayed with images. All approved of the colour of the bowl and spoon, saying that because they are white 'dirt can be easily detected', thereby promoting cleanliness. There was also high demand for the tools in communities where user testing occurred. During the post user-testing FGD a rural mother said:

'I think if people in my area are given, most of them will use it because they keep asking me questions and ask me why I am the only one who got it. They say that I should have informed them when they [the tools] were being distributed so that they [the neighbours] go and get one too. I tell them that it is by the Grace of God that I was chosen to be given one. They said that I should inform them if I hear that the bowls are being distributed; and I told them that I definitely will.'

Several women indicated that they were requested by family members and neighbours to teach about the bowl and its benefits and that these mothers marked their own household feeding cups and bowls using the feeding bowl as a guide:

'The first time it was my co-wife who came and asked what it was; I told her it was a bowl for feeding the baby any food that I have. Then she asked if the bowl would make the baby full, and 1 told her that 1 was given [the bowl] because it shows the amounts that 1 should feed the baby. Then she asked me to show her [the mark] and 1 did.' (Urban mother of a 7-month-old)

'One woman told me that if I was living near her, she would come borrow the bowl and use it to measure food and mark the correct levels on her cup so that she can know the correct amount of food to feed the baby.' (Rural mother of a 14-month-old)

'[Neighbours] asked me questions.'

Interviewer: 'What questions did they ask?'

Mother: 'What amount of food do the people that brought these tools want the babies to eat?... I explained it to them using the card.' (Rural mother of a 7-month-old)

\section{Discussion}

Our work found that a simple set of dietary tools consisting of a demarcated bowl, slotted spoon and illustrated 
counselling card are feasible and acceptable tools that can cue optimal dietary practices of mothers during pregnancy, the first 6 months postpartum and children aged 6-18 months. Mothers reported that they consumed more food, that children were fed greater amounts of thicker and more diverse foods, and that meal frequency increased with use of the tools. As well, pregnant women reported weight gain and improved health, breast-feeding women cited increased milk production and mothers with older children reported improved infant health, energy and growth. However, it should be noted that these benefits were perceived as we did not quantitatively assess anthropometry, infant health or breast milk output.

Our work is consistent with the Manoff Group which found a similar demarcated bowl for complementary feeding of children aged 6-24 months was well accepted by communities ${ }^{(22)}$. While the Manoff bowl used in Latin America is an acceptable tool to cue appropriate meal volume for children aged 6-24 months, our set of tools are the first of their kind to cue optimal diets through the entire first 1000 days of life. Furthermore, they target additional, often difficult-to-conceptualize recommendations for maternal and child nutrition, including extra food requirements during pregnancy and breast-feeding, appropriate meal frequency and appropriate thickness of infant porridge. Formative testing of similar tools by our team in Bihar, India yielded similar positive findings with regard to acceptability to improve maternal and infant dietary practices during the first 1000 days $^{(23)}$.

We hypothesize that the tools improved practices and perceived health through clarification of recommendations made during counselling and enhanced self-efficacy. Lack of knowledge or confusion about amounts, frequency and diversity of complementary feeding has previously been reported as a challenge to achieving optimal intakes among young children ${ }^{(24,25)}$. Mothers attributed changes in their practices to their use of the tools and the increase in their confidence to feed their children the appropriate amount, consistency and frequency of foods. We hypothesize that participating mothers were empowered by the bowl demarcations and counselling card because they bridged the gap between families' desire to feed their children optimal amounts and the knowledge and skills required to do so. The positive perceived benefits related to mothers' and infants' health and growth that resulted from implementing the recommended dietary practices may have served as 'affirmations of competence' that contributed to self-efficacy with the recommended practice ${ }^{(26)}$. These intrinsic cues could potentiate continued practice of the behaviour and ultimately contribute to sustained behaviour change.

Some breast-feeding women perceived increases in milk production and attributed these increases to the additional food they were consuming as a result of using the tools. They cited greater confidence in their ability to exclusively breast-feed, with some claiming they had switched from mixed feeding back to exclusive breastfeeding or were intending to delay other foods as a result of using the tools. Several authors have written about maternal perceptions of food consumption and its association with milk production ${ }^{(27-30)}$. In a mixed-methods study in Nakuru, Kenya, Webb-Girard et al. observed a strong positive conceptual link between maternal hunger and food insecurity and the perceived ability to produce sufficient breast milk ${ }^{(31)}$. An increased perceived ability to produce sufficient milk may increase breast-feeding selfefficacy, a critical behavioural component for successful breast-feeding $^{(32)}$.

\section{Facilitators and barriers to uptake}

According to the social-ecological model of behaviour change, an individual's behaviours are affected by relational and structural factors that can undermine or support the uptake of new ideas ${ }^{(33,34)}$. We identified several environmental and social factors that influenced tool uptake and should be considered in future work or in adaptation to other contexts. The recommendation for breast-feeding women to consume an extra meal each day was congruent with cultural norms and thus this recommendation was accepted with ease when food was considered available. However, in situations of food insecurity, women in our study said they felt they could not consume extra food. Interestingly food insecurity was not reported as barrier to increased child food consumption; this may be due to the reported cultural expectations for mothers to eat last during times of food scarcity as a way to ensure enough food for children. However, this coping strategy may be less effective if food insecurity persists or deepens. Indeed, in a meta-analysis of interventions to improve complementary feeding practices and child growth, Dewey and Adu-Afarwuah identified food insecurity as a significant modifier of impact ${ }^{(5)}$. In contexts with high food insecurity, nutrition and education strategies to improve complementary feeding were less effective than those in food-secure contexts. Such findings speak to the need to address the distal determinants of nutrition related to availability and accessibility of nutritious foods.

In our study context, fears of choking and beliefs about the ill effects of certain foods were a prominent reason for not feeding nutritious foods to young children. We found counselling about food diversity and frequency was more acceptable because children consumed the recommended foods when offered to them, but increasing thickness of porridge remained a challenge due to fear of choking. Numerous studies in multiple contexts have documented food taboos and fears related to choking and illness as commonly cited barriers to feeding nutrient-dense foods, such as animal-source foods and legumes, to young children $^{(11,35)}$. However, what has received less attention is the development of effective strategies to overcome or shift taboos such that these foods become acceptable. As part of 
user testing, research assistants used participatory strategies that promote dialogue about dietary practices and underlying beliefs and where requested conducted feeding demonstrations with families to show what children can safely eat, and included multiple family members in this dialogue. We hypothesize that the participatory inclusion of influential family members in counselling dialogues was important for acceptance and uptake, thus the inclusion of family members in counselling efforts should be encouraged. However, we acknowledge that the feasibility of integrating the time- and resource-intensive counselling strategies used in the present research into larger-scale counselling efforts with the tools should be explored.

The recommendation to consume more food during pregnancy conflicted with cultural norms and thus this recommendation was more difficult for pregnant women to put into practice. Similar findings regarding eating down have been reported by Christian et al. who found that Nepalese women not only ate less frequently, but also limited the diversity of foods consumed during pregnancy $^{(36)}$. Women ate less for various reasons including personal preference, seasonal availability and the desire for smaller babies to ease delivery ${ }^{(36)}$. In contexts where home deliveries are common and health systems' capacity for managing complications during delivery is inadequate, this cultural norm towards eating down likely is a protective strategy against pregnancy complications and maternal morbidity and mortality. It therefore deserves careful consideration when developing nutrition behaviour change strategies for pregnancy.

\section{Limitations and future research}

Despite the positive findings from the present research there are limitations. First, the study was formative in nature and designed to qualitatively test acceptability and feasibility of the tools, including gain insights into community recommendations for design improvements. As a result, the sample size was small, the user testing period was short and dietary changes were assessed qualitatively. Perceived benefits related to changes in maternal and child appetite, health and weight were based on self-reports and not confirmed quantitatively with anthropometry, quantitative $24 \mathrm{~h}$ dietary recalls or morbidity recalls. The counselling provided during user testing occurred thrice in a $28 \mathrm{~d}$ period and was provided by university graduates with degrees in nutrition. Thus the counselling sessions provided in the study are likely more time- and resource-intensive than what would be provided as part of a standard nutrition programme or a package of community health worker responsibilities. Because of the simultaneous implementation of intense and frequent counselling with provision of the tools, we are unable to say whether the tools or the intensive counselling or a combination of the two resulted in the reported improvements. It would be useful to test the effectiveness of the tools when administered with counselling intensity that better reflects standard practice.
In light of these limitations, future research should clarify the efficacy and effectiveness of the tools utilizing quasiexperimental or randomized controlled designs. Quantitative indicators of impact including changes in dietary practices (diversity, consistency and frequency), micronutrient and macronutrient intakes, and anthropometry are essential. Mixed-methods approaches should be utilized to also capture the effects of the tools on domains of behaviour change including beliefs and self-efficacy. Given that there are costs associated with use of the tools compared with counselling alone, cost-effectiveness research is critical to inform programming decisions regarding the most important tools. Lastly, research is needed to identify effective and efficient community-based delivery platforms. For example, the current concept for the tools is family centred: they are to be provided to and used by families to cue dietary practices. However, it is plausible that the tools could be equally as effective if used as a job aide for community health workers to improve their counselling efforts without the added expense of providing tools to all families. The Alive and Thrive Program in Bangladesh and CARE's Integrated Family Health Initiative in Bihar, India are using marked katoris (steel bowls) as job aides for community health workers to improve counselling on complementary feeding; however, evaluations of this approach are not available. It would be useful to document the relative effectiveness and cost-effectiveness of providing the tools to families $v$. their use as a health worker job aide.

In summary, a demarcated bowl, slotted spoon and illustrated counselling card were acceptable and feasible tools for cueing optimal dietary practices of mothers and infants in Western Kenya. Their use as a cue to action may significantly enhance existing behaviour change communication and counselling strategies that aim to improve nutrition during the first 1000 days.

\section{Acknowledgements}

Acknowledgements: The authors would like to acknowledge Lenette Golding, PhD, Alyssa Lowe, MPH and Ann Digiralamo, $\mathrm{PhD}$ for their valuable inputs on the toolkit and applications for nutrition programming and social and behaviour change. They also thank the research assistants, including Irene Adema, Christine Imali, Janerose Lusweti, Daisy Mukoya, Irene Namai, Catherine Serange and Faith Tonui, and all of the participants, with special thanks to women who participated in user testing. Financial support: This project was funded by Grand Challenges Explorations (grant number OPP1058623), the Bill and Melinda Gates Foundation. N.K. was sponsored by the Emory Global Health Institute Individual Field Scholars Program. The funders had no role in the design, analysis or writing of this article. Conflict of interest: None. Authorship: N.K. developed data collection tools, managed data collection, completed data analysis, drafted 
the article and contributed to revisions; S.M. provided design input on the bowl, spoon and counselling card (i.e. toolkit), managed data collection and contributed to manuscript development; E.K. contributed to design of the counselling cards, developed field protocols and managed data collection; D.K.C. developed data collection tools and provided design input on the toolkit; J.C. provided design inputs and oversaw prototyping of the bowl and spoon; W.B. provided design input on the toolkit; F.G. was the in-country field mentor; A.W.G., Principal Investigator, contributed to all aspects of the toolkit design and development, the research protocol and the development of data collection tools, data analysis, and manuscript preparation and revisions; all authors reviewed the article and provided input on the final draft. Ethics of buman subject participation: This study was conducted according to the guidelines laid down in the Declaration of Helsinki and all procedures involving human subjects were approved by Emory University and the Kenyan Medical Research Institute ethical review boards. All participants provided informed consent.

\section{References}

1. Bhutta ZA, Das JK, Rizvi A et al. (2013) Evidence-based interventions for improvement of maternal and child nutrition: what can be done and at what cost? Lancet 382, 452-477.

2. Fabrizio CS, van Liere M \& Pelto G (2014) Identifying determinants of effective complementary feeding behaviour change interventions in developing countries. Matern Child Nutr 10, 575-592.

3. Girard AW \& Olude O (2012) Nutrition education and counselling provided during pregnancy: effects on maternal, neonatal and child health outcomes. Paediatr Perinat Epidemiol 26, Suppl. 1, 191-204.

4. Haroon S, Das JK, Salam RA et al. (2013) Breastfeeding promotion interventions and breastfeeding practices: a systematic review. BMC Public Health 13, Suppl. 3, S20.

5. Dewey KG \& Adu-Afarwuah S (2008) Systematic review of the efficacy and effectiveness of complementary feeding interventions in developing countries. Matern Child Nutr $\mathbf{4}$, Suppl. 1, 24-85.

6. Pelto GH, Martin SL, van Liere MJ et al. (2015) Perspectives and reflections on the practice of behaviour change communication for infant and young child feeding. Matern Child Nutr (Epublication ahead of print version).

7. Champion VL \& Sugg Skinner C (2008) The Health Belief Model. In Health Behavior and Health Education: Theory, Research, and Practice, 4th ed., pp. 46-65 [K Glanz, BK Rimer and K Viswanath, editors]. San Francisco, CA: Jossey-Bass.

8. Orji R, Vassileva J \& Mandryk R (2012) Towards an effective health interventions design: an extension of the health belief model. Online J Public Health Inform 4, 3.

9. Shannon K, Mahmud Z, Asfia A et al. (2008) The social and environmental factors underlying maternal malnutrition in rural Bangladesh: implications for reproductive health and nutrition programs. Health Care Women Int 29, 826-840.

10. Imdad A, Yakoob MY \& Bhutta ZA (2011) Effect of breastfeeding promotion interventions on breastfeeding rates, with special focus on developing countries. BMC Public Health 11, Suppl. 3, S24.
11. Pemunta NV \& Fubah MA (2014) Socio-cultural determinants of infant malnutrition in Cameroon. J Biosoc Sci $\mathbf{4 7}$, 423-448.

12. Santos-Torres MI \& Vasquez-Garibay E (2003) Food taboos among nursing mothers of Mexico. J Health Popul Nutr 21, 142-149.

13. Lutter CK, Daelmans BM, de Onis M et al. (2011) Undernutrition, poor feeding practices, and low coverage of key nutrition interventions. Pediatrics 128, e1418-e1427.

14. Black RE, Victora CG, Walker SP et al. (2013) Maternal and child undernutrition and overweight in low-income and middle-income countries. Lancet 382, 427-451.

15. Manoff Group (2009) The Child Feeding Bowl. Global Health Council Conference. http://www.manoffgroup.com/ documents/ChildFeedingBowlPowerpointfinal0509.pdf (accessed September 2015).

16. Food and Nutrtion Infomation Center, US Department of Agricuture (2011) Dietary Reference Intakes: Estimated Average Requirements and Recommended Intakes. http:// fnic.nal.usda.gov/dietary-guidance/dietary-reference-intakes/ dri-tables (accessed May 2014).

17. Dewey KG \& Brown KH (2003) Update on technical issues concerning complementary feeding of young children in developing countries and implications for intervention programs. Food Nutr Bull 24, 5-28.

18. Kenya National Bureau of Statistics, Ministry of Health, National AIDS Control Council et al (2015) Kenya Demographic and Health Survey 2014: Key Indicators. Nairobi: KNBS.

19. Black RE, Allen LH, Bhutta ZA et al. (2008) Maternal and child undernutrition: global and regional exposures and health consequences. Lancet 371, 243-260.

20. Kenya National Bureau of Statistics \& ICF Macro (2010) Kenya Demographic and Health Survey 2008-09. Nairobi and Calverton, MD: KNBS and ICF Macro.

21. Okon'go O, Kisia A \& Odongo DO (2012) Dietary iron status and health of third trimester pregnant women. Food Sci Qual Manage 4, 24-29.

22. Ali D, Saha KK, Nguyen PH et al. (2013) Household food insecurity is associated with higher child undernutrition in Bangladesh, Ethiopia, and Vietnam, but the effect is not mediated by child dietary diversity. J Nutr $\mathbf{1 4 3}$, 2015-2021.

23. Collison DK, Kekre P, Verma P et al. (2015) Acceptability and utility of an innovative feeding toolkit to improve maternal and child dietary practices in Bihar, India. Food Nutr Bull 36, 24-32.

24. Paul KH, Muti M, Chasekwa B et al. (2012) Complementary feeding messages that target cultural barriers enhance both the use of Lipid-Based Nutrient Supplements and underlying feeding practices to improve infant diets in rural Zimbabwe. Matern Child Nutr 8, 225-238.

25. Sanghvi T, Jimerson A, Hajeebhoy N et al. (2013) Tailoring communication strategies to improve infant and young child feeding practices in different country settings. Food Nutr Bull 34, issue 3, 169S-180S.

26. Deci EL, Koestner R \& Ryan RM (1999) A meta-analytic review of experiments examining the effects of extrinsic rewards on intrinsic motivation. Psychol Bull 125, 627-668.

27. Wright CM, Parkinson KN \& Drewett RF (2004) Why are babies weaned early? Data from a prospective population based cohort study. Arch Dis Child 89, 813-816.

28. Sorensen E, Fernando DN, Hettiarachchi I et al. (1998) Exclusive breastfeeding among women on the plantations in Sri Lanka. J Trop Pediatr 44, 313-315.

29. Cherop CE, Keverenge-Ettyang AG \& Mbagaya GM (2009) Barriers to exclusive breastfeeding among infants aged 0-6 months in Eldoret Municipality, Kenya. East Afr J Public Health 6, 69-72. 
30. Gonzalez-Cossio T, Habicht JP, Rasmussen KM et al. (1998) Impact of food supplementation during lactation on infant breast-milk intake and on the proportion of infants exclusively breast-fed. J Nutr 128, 1692-1702.

31. Webb-Girard A, Cherobon A, Mbugua S et al. (2012) Food insecurity is associated with attitudes towards exclusive breastfeeding among women in urban Kenya. Matern Child Nutr 8, 199-214.

32. Blyth R, Creedy DK, Dennis CL et al. (2002) Effect of maternal confidence on breastfeeding duration: an application of breastfeeding self-efficacy theory. Birth $\mathbf{2 9}$, 278-284.
33. Stokols D (1996) Translating social ecological theory into guidelines for community health promotion. Am J Health Promot 10, 282-298.

34. Glanz KR, Rimer BK \& Viswanath K (editors) 2008) Health Behavior and Health Education: Theory, Research, and Practice, 4th ed. San Francisco, CA: Jossey-Bass.

35. Meyer-Rochow VB (2009) Food taboos: their origins and purposes. J Ethnobiol Ethnomed 5, 18.

36. Christian P, Srihari SB, Thorne-Lyman A et al. (2007) Eating down in pregnancy: exploring food-related beliefs and practices of pregnancy in rural Nepal. Ecol Food Nutr $\mathbf{4 6}$, 253-278. 